Lun 10

NON SMALL CELL LUNG CANCER :FEASIBILITY OF CONCOMITANT IRRADIATION AND CHEMOTHERAPY. J.H.Karstens, D.Bremer, A.Pohling, J.Ammon

Fatients (pts) with non small cell lung cancer (NSCLC) with local extension and/or mediastinal involvement (stage III,UICC) still carry a particular poor prognosis. Short median survival time (MST) is a strong argument to add chemotherapy (CT) to radiotherapy (RT) with the intention of an acceptable total treatment time. We performed three feasibility studies with RT and concomitant $\mathrm{CT}$ and chose cis-Platinum (cP) or vindesine (V) as single agents and in a third study $\mathrm{RT}+\mathrm{CP}+\mathrm{V}$. 16 previously untreated pts with NSCLC entered a study with simultaneous RT with CP. RT consisted in a median dose of 45 Gy; $c P$ was administered in all pts as 120 hrs infusion ( $20 \mathrm{mg} / \mathrm{gm} / 24 \mathrm{hrs}$ ) during week 1,4 and 7. Continuous infusion has been reported to be less toxic and at least as effective as cP given by bolus. CR was observed in 3 , and $P R$ in 8 pts. Considering only stage III pts $(n=10)$ MST is 11 months. From this study we concluded: concomitant RT with $\mathrm{CP}$ is feasible without major radiation toxicities; an increase of RT dose appears possible. Since 1/85 we started a protocol with 55-60 Gy RT and $\mathrm{CP}(20 \mathrm{mg} / \mathrm{gm} / 24 \mathrm{hrs}$ for 100 hrs) during week 1,4 and 7 and weekly bolus of $3 \mathrm{mg} / \mathrm{qm} V$ during week $2,3,5,6$ and 8 . In a third study, pts> 65 years were treated with $V$ as a weekly bolus during $\sigma$ weeks without $\mathrm{cP}$. Unpublished data of an ongoing randomized trial of the Southeastern Cancer Study Group suggests a better local response. Preliminary results with our two protocols, RT $+\mathrm{CP}+\mathrm{V}$ (10 pts) and RT $+V$ (10 pts) are promising without major toxicities.

Abt. Strahlentherapie,Med.Fakultät,Klinikum RWTH Aachen, D - 5100 Aachen, Pauwelsstr.

\section{Lun 11}

CHEMOTHERAPY OF ADUANCED NON-SMALL-CELL LUNG CANCER WITH DDP AND HIGH-DOSE ETOPOSIDE

U. Gatzemeier, D.K. Hossfeld, H. Magnussen, D. Radenbach, R. Zschaber

Introduction: Cis-Platinum and VP-16 are active agents in the treatment of NSCLC with synergism in experimental models. A dose-finding study of VP-16 with a fixed dose of DDP led us to conclude that the maximally tolerated dose i.v. of VP-16 was in the range of $500 \mathrm{mg} / \mathrm{m}^{2}$ per course. To define the activity and toxicity we started a phase-IItrial.

Patients and methods: Since 8/1983 41 patients with untreated inoperable NSCLC were entered into the protocoll until 3/84. 40 patients were evaluable for response and median survival time. Patients characteristics: $75 \%$ ED. Median age 57 years. Perfarmance-Status more than $70 \%$ in all patients. Histology: 23 squamous cell, 9 large cell, 9 adeno carcinomas.

Dose schedule: DDP $100 \mathrm{mg} / \mathrm{m}_{2}^{2}$ day 1

$$
\text { UP-16 } 170 \mathrm{mg} / \mathrm{m}^{2} \text { day } 3 / 4 / 5
$$

Results: The response rate twas $16 / 40$ patients (40\%). $\overline{2}$ patients ( $5 \%$ ) achieved a complete remission, $14 \mathrm{pa}-$ tients ( $35 \%$ ) a partial remission. The median survival time in responders is 13 months (follow up 16 months) including 3 early drug related deaths, in non-responders 8 months. The toxicity of the chemotherapy was a serious problem, 4 patients achieved a severe agranulocytosis, which leads to 3 drug related early death with septic fever.

Conclusion: The combination DDP and high-dose VP-16 did not improve the results of response and median survival time in NSCLC in retrospective comparison to regime with lower dosages of these drugs (own results). The side effects however are much more serious, especially the hematological toxicity. Therefore these dosages can't be recommanded in the treatment of patients with inoperable NSCLC.

Dep. of Pneumological Oncology Großhansdorf Hospital Wöhrendamm 80, 2070 Großhansdorf

\section{Lun 12}

HYPERFRACTIONATED RADIOTHERAPY OF BRONCHOGENIC CARCINOMA W. Berberich, K. Schnabel, B. Scharding, H.J.Tkocz, M. Niewald, C.Beck, P.Schl.immer We have irradiated 100 patients with small cen (SCLC) and non small cell lung cancer (NSCLC). 41 patients were treated twice daily and 59 with a conventional fractionation scheme. Patients with SCLC were irradiated either with 52 Gy/40 fractions/4 weeks in a hyperfractionated way or with 46 Gy/23 fractions $/ 4.3$ weeks. Patients with NSCLC received either 65 Gy $/ 50$ fractions $/ 5$ weeks (hyperfractionated schedule) or $60 \mathrm{~Gy} / 30$ fractions $/ 6$ weeks (conventional regimen). Concerning age distribution, tumor stage, Karnofsky index and previous treatment the respective groups were well balanced. In the case of patient with NSCLC life table (Kaplan-Meier estimate) showed no difference between the therapy schedules. Median survival was approx. $330 \mathrm{~d}$. The treatment groups were not different with respect to progression-free interval and occurence of distant metastases. Concerning SCLC the KaplanMeier estimate of survival showed no significant difference between both groups, but in the hyperfractionated arm the results seem to be somewhat better. Median survival was $330 \mathrm{~d}$ after conventional treatment and exceeded $450 \mathrm{~d}$ after hyperfractionation. We found no difference concerning the occurence of distant metastases. Radiation pneumonitis occured in all groups but significant differences could not be seen. We therefore believe that we have no underdosage with the hyperf ractionated regimens and that these schemes could be of some value treating rapidly proliferating tumors.

Abt. für Strahlentherapie, Radiol. Universitätsklinik D-6650 Homburg/Saar

\section{Lun 13}

THE NEW ANTHRACYCLINE THP-ADM (4' - O-TETRAHYDROPYRANYLADRIAMYCIN) IN THE TREATMENT OF MALIGNANT PLEURAMESOTHELIOMAS

J. Gatzemeier, A. Calvrezos, E. Kaukel, G. Koschel, H. Magnussen, D. Radenbach

Introduction: In the treatment of pleuramesatheliomas some drugs seem to be active. Doxorubicin as single agent is the most active drug reaching a response rate of approximately $40 \%$ (Vogelzang 1984). The efficacy of the new anthracycline-analogue THP-ADM is similar or better to Adriamycin but the side effects are less pronounced. To assess the effectiveness and side effects we started a phase-II-trial.

Patients and Methods: Since $10 / 198313$ patients with histologically proven pleuramesotheliomas were entered into the protocoll. 7 male/6 female with a median age of 57 years. The performance status of all patients was more than $70 \%$.

Dose schedule (Majima): $40-50 \mathrm{mg} / \mathrm{m}^{2}$ i.v. bolus every 3 weeks.

Results: The response rate was $1 / 13(7,6 \%$ partial remission.

$7 / 13$ (53,8 \%) patients showed stable disease.

$5 / 18(38,4 \%)$ had progressive disease.

The median survival time with a minimum observation period of 7 month was 9,5 month. In general, the side effects were extremely mild; we didn't see any severe cardiotoxicity. Gastrointestinal side effects are very rare. In summary, the antitumor effect of THP-ADM as single agent in pleuramesotheliomas is only low. But the maximally talerated dose appears to be much higher than 40 $50 \mathrm{mg} / \mathrm{m}^{2}$ per course, In the following trial we were going to use $70-75 \mathrm{mg} / \mathrm{m}^{2}$ per course with still acceptable toxicity in the first 3 patients.

Dep. of Pneumological Oncology Großhansdorf Hospital Wöhrendamm 80, D-2070 Großhansdorf 